\title{
Intravesical thermometer: an unusual complication of vaginal temperature measurement
}

\author{
P J Riou, A Harris
}

A 28 year old nulliparous woman presented to the accident and emergency department after failing to retrieve a glass mercury thermometer from her vagina. She had been using the thermometer to measure her vaginal temperature on waking each morning as part of a fertility programme in order to ascertain the time of ovulation. Although oral or rectal temperature measurements are usually used to estimate core body temperature, in this instance the patient's general practitioner had recommended vaginal measurement as an acceptable alternative. In addition, specific popular health care books obtained by the patient had also recommended vaginal temperature measurements.

Initially digital and speculum examinations of her vagina were carried out-both were unremarkable. A plain radiograph of the pelvis was obtained. This clearly demonstrated the thermometer lying transversely within the pelvis (fig 1). Subsequent ultrasonography of the pelvis showed the thermometer within the urinary bladder. The patient was referred to the duty urologist who removed the thermometer via a rigid cystoscope under general anaesthesia without complication.

This is an extremely unusual case where a patient has passed a mercury thermometer per

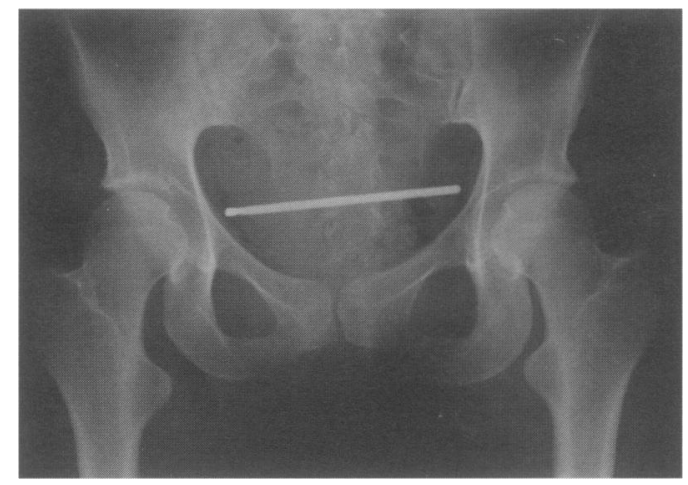

Figure 1 Thermometer shown lying within the pelvis.

urethram into her bladder while attempting to obtain a vaginal temperature measurement. Although the vagina is an accepted site in which temperature readings may be obtained, it is quite clear that this case could have been avoided had oral or rectal measurements been preferred. In addition there can be no doubt that if, as suggested, measurements are taken immediately on waking before the patient is fully alert, misplacement of the thermometer is much more likely.

\section{Infected chronic extradural haematoma}

\author{
Andrew R J Mitchell, Kassem S El-Shunnar
}

A 17 year old man was referred to Torbay Hospital with a two week history of headaches. These were frontal and bitemporal in location,

\author{
Clinical Fellow in \\ Cardiology, Royal \\ Cornwall Hospital, \\ Truro, Cornwall \\ TR1 3LJ \\ A R J Mitchell \\ Consultant \\ Neurosurgeon, \\ Derriford Hospital, \\ Plymouth, Devon \\ K S El-Shunnar

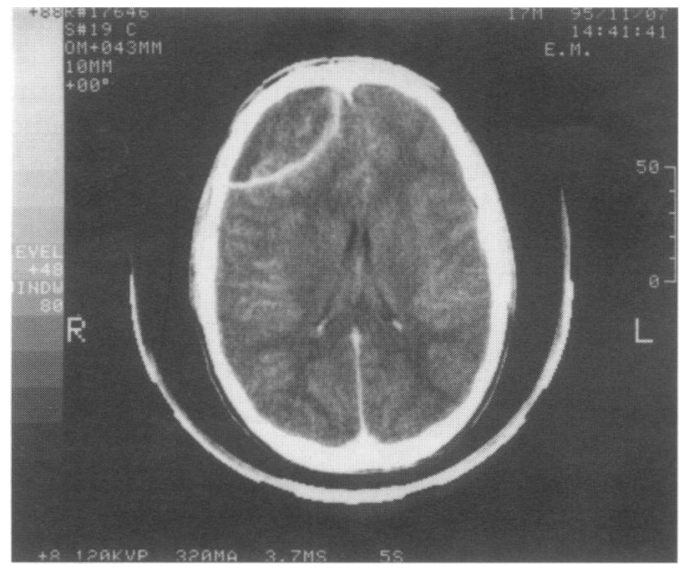

Figure 1 Computed tomogram of the patient's head. worse on coughing and leaning forward, and with a clear pattern of increasing frequency and severity. There was no history of trauma, no history of nausea or visual disturbance, and no family history of migraines. Physical examination revealed no evidence of meningism or localising neurological signs but fundoscopy showed slight blurring of the disc margins bilaterally. The patient was apyrexial and had a small tender soft tissue swelling over the right forehead. He was admitted for observation and investigation but soon after admission his headaches worsened and he started to vomit. Computed tomography of his head was performed urgently and revealed a calcified chronic right frontal extradural collection with some mass effect (fig 1). He was referred for urgent surgery at Derriford Hospital, Plymouth and underwent emergency frontal craniectomy with drainage of an infected extradural haematoma. Streptococcus anginosus was isolated from the 